\title{
Editorial: Building Quantum Networks
}

From the Internet and the power grid to financial markets and the brain itself, networks are critical to the behavior of complex systems [1]. Broadly defined, a network is a set of nodes, which can contain some processing capability connected by channels, along which information or energy flows.

Over the past decade, there has been rapid progress in developing quantum technologies: new kinds of devices that harness the quantum phenomena of superposition and entanglement to build better sensors, to guarantee information security, or to enlarge the class of computations that can be performed efficiently. Extending networks to the quantum domain will provide a useful framework for implementing and expanding these technologies.

Network structures have a number of features that are useful for quantum information. Quantum machines are fiendishly difficult to build, and it is consequently easier to construct simple ones-physical systems encoding just a few quantum bits (or qubits) - than more complex ones. Thus, networks are less demanding on putative quantum manufacturing capabilities: it is only necessary to construct one type of system for a node, one for a channel, and reproduce these simple components across the network.

Further, increasing the scale of a network to increase capacity and performance does not require a radical change in architecture. Finally, networks can be robust to imperfections: they may be dynamically reconfigured, and poorly operating elements can be circumvented or replaced. The powerful principles of manufacturability, scalability, and robustness underpin the role of networks as the primary means by which we process, consume, and communicate information in the modern world. These same features enable networks to provide solutions for universal quantum communications, simulators, and even computers.

A quantum network [2] inherits the features of robustness and scalability from its classical network counterparts, but because each node and channel has quantum capability, the overall network can achieve things that are effectively impossible with conventional technologies. Elementary quantum systems can be connected together to form flexible, scalable solutions for quantum-enhanced computing [3], advanced simulation [4], secure communications [5], and distributed sensing [6]. For some applications, quantum-enhanced systems are expected to improve on the best classical performance by factors that grow exponentially with the network size. For instance, fully universal quantum computers can factor large numbers that any foreseeable computer designed around classical physics cannot. This capability will render the most popular method for data encryption vulnerable to attack, spurring further research into quantum-enhanced algorithms.

In other applications, such as device-independent cloud computing [7] and unconditional security in communications [8], completely new features, with no classical analogues, appear, such as the ability to guarantee the secrecy of information by means of the laws of physics, rather than by trusted delivery.

Universal quantum computing machines are still a long way off, but more specialized machines that can perform specific tasks are much closer. Some of these are termed quantum simulators because they are designed to simulate particular physical systems. These devices may be used to design new materials or drugs, or to optimize logistics.

Hybrid light-matter networks, in which the channels use light as a communication medium and the nodes use matter for processing operations, have emerged as a particularly important class because they make best use of the inherent properties of each of those media. Light can carry high-bandwidth quantum signals over long distances in ambient conditions. One reason that this is possible is that photons do not interact with each other easily, and, thus, information remains uncorrupted provided the photons are not scattered out of the channel or absorbed.

This lack of interaction is a liability though, when you need to process information, which inevitably means that you must change the state of one physical system in response to the state of another. Matter is, therefore, used to construct the best quantum logic elements: for instance, atoms and ions can interact by means of [9] Coulomb forces in a trap. This makes it possible to operate quantum logic gates in which the state of one ion changes deterministically in response to the state of another. Further, atoms and ions can emit and absorb light efficiently. 
For this reason, hybrid atom-light architectures have been advocated as a means to build longdistance quantum communications networks [10] as well as to provide the most promising architecture for a quantum computer $[9,11,12]$.

Promising though hybrid quantum networks are, several challenges remain. A first challenge is that such a network cannot make use of an optical amplifier. This is because amplifiers for arbitrary quantum signals inevitably add noise, and noise ultimately masks the quantum correlations that are at the heart of enhanced information processing.

Another challenge is that in quantum networks, operations are sometimes nondeterministic. For instance, some operations may be conditioned on the outcome of a measurement of one of its constituent quantum systems. This necessarily produces a result at random: this is just the nature of quantum measurement. Noise and indeterminate measurement militate against the idea of scalability because the successful operation of the network relies on all individual local operations being completed successfully at the same time. The probability of the network succeeding falls exponentially as the number of local operations increases if they are not deterministic.

So how can you enlarge a network whose nodes operate randomly or noisily? The answer is to use quantum memories. A quantum memory is a device that can store the outcome of a quantum operation [13]. In particular, in a hybrid light-matter network, a quantum memory is a material system that can store and retrieve quantum light on demand. That is, the application of a control pulse enables the quantum signal to be "written" into the memory, and a subsequent control pulse "reads" out the light. Thus, a quantum memory enables scalable networks by holding onto the state produced by one node of a network until the next node has completed its operation. That way, the time taken to prepare the network decreases from being exponential in the number of nodes to being a polynomial function of that number [14].

Generating quantum correlations between nodes of a network is perhaps the most obvious and primary function of a quantum memory, but they can also be used as active elements, for example, as frequency shifters or quantum drop-add filters for mode-selective multiplexing, as well as having the potential for implementing deterministic entangling operations.

The basic requirements of a quantum memory seem, on the face of it, to be benign. It is well known how to hold onto single atoms or ions and to couple light in and out of them efficiently, so surely it ought to be possible to use these as storage medium components. Alternatively, lowloss optical fibers are available: could they be used to delay quantum light pulses as other operations take place elsewhere in the network? Indeed, both of these possible solutions are being pursued by research groups around the world.

Nonetheless, efficient light-matter coupling in a scalable device remains a technical challenge because component performance (e.g., low-loss, fast optical switches) and ease of fabrication and operation (e.g., ambient-temperature storage media) are not yet available.

For this reason, it has proven difficult to prepare entangled states of multiple nodes of an extended network or indeed to synchronize two or more nodes by storing light in matter. Because of these technical challenges, only a few laboratory-scale experiments have succeeded in making efficient and low-noise quantum memories.

Nevertheless, the technical challenges are gradually being overcome. We anticipate that in the next few years there will be a variety of options for manufacturable quantum memories that can be built simply and cheaply, and this will be a tipping point for quantum networking.

The rapid progress in this field portends a functional quantum network in the next decade that will open the door to a number of different applications, both long awaited and unanticipated. Hold onto your photons.

Published 21 October 2016

Ian A. Walmsley and Joshua Nunn

Networked Quantum Information Technologies Hub

Department of Physics

University of Oxford, Clarendon Laboratory

Parks Road, Oxford OX1 3PU, United Kingdom

DOI: 10.1103/PhysRevApplied.6.040001 
[1] Réka Albert and Albert-László Barabási, Statistical mechanics of complex networks, Rev. Mod. Phys. 74, 47 (2002).

[2] H. J. Kimble, The quantum internet, Nature (London) 453, 1023 (2008).

[3] Ying Li and Simon C. Benjamin, Hierarchical surface code for network quantum computing with modules of arbitrary size, arXiv:1509.07796.

[4] E. Rico, T. Pichler, M. Dalmonte, P. Zoller, and S. Montangero, Tensor Networks for Lattice Gauge Theories and Atomic Quantum Simulation, Phys. Rev. Lett. 112, 201601 (2014).

[5] Bernd Fröhlich, James F. Dynes, Marco Lucamarini, Andrew W. Sharpe, Zhiliang Yuan, and Andrew J. Shields, A quantum access network, Nature (London) 501, 69 (2013).

[6] Jung-Shian Li and Ching-Fang Yang, in IEEE 6th International Conference on Mobile Adhoc and Sensor Systems, Macau, 2009, pp. 1024-1029, http://ieeexplore .ieee.org/document/5337016/.

[7] Alexandru Gheorghiu, Elham Kashefi, and Petros Wallden, Robustness and device independence of verifiable blind quantum computing, New J. Phys. 17, 083040 (2015).

[8] Ioannis Kogias, Yu Xiang, Qiongyi He, and Gerardo Adesso, Unconditional security of entanglement-based quantum secret sharing schemes, arXiv:1603.03224.
[9] W. J. Munro, K. A. Harrison, A. M. Stephens, S. J. Devitt, and Kae Nemoto, From quantum multiplexing to highperformance quantum networking, Nat. Photonics 4, 792 (2010).

[10] Stephan Ritter, Christian Nölleke, Carolin Hahn, Andreas Reiserer, Andreas Neuzner, Manuel Uphoff, Martin Mücke, Eden Figueroa, Joerg Bochmann, and Gerhard Rempe, An elementary quantum network of single atoms in optical cavities, Nature (London) 484, 195 (2012).

[11] Sean D. Barrett and Pieter Kok, Efficient high-fidelity quantum computation using matter qubits and linear optics, Phys. Rev. A 71, 060310 (2005).

[12] L.-M. Duan and C. Monroe, Colloquium: Quantum networks with trapped ions, Rev. Mod. Phys. 82, 1209 (2010).

[13] C. Simon, M. Afzelius, J. Appel, A. Boyer de la Giroday, S. J. Dewhurst, N. Gisin, C. Y. Hu, F. Jelezko, S. Kroll, J. H. Muller, J. Nunn, E. Polzik, J. Rarity, H. de Riedmatten, W. Rosenfeld, A. J. Shields, N. Skold, R. M. Stevenson, R. Thew, I. Walmsley, M. Weber, H. Weinfurter, J. Wrachtrup, and R. J. Young, Quantum memories, Eur. Phys. J. D 58, 1 (2010).

[14] L.-M. Duan, M. D. Lukin, J. I. Cirac, and P. Zoller, Longdistance quantum communication with atomic ensembles and linear optics, Nature (London) 414, 413 (2001). 